\title{
Selective Regulation of Current Densities Underlies Spontaneous Changes in the Activity of Cultured Neurons
}

\author{
Gina Turrigiano, Gwendal LeMasson, ${ }^{a}$ and Eve Marder \\ Department of Biology and Center for Complex Systems, Brandeis University, Waltham, Massachusetts 02254
}

We study the electrical activity patterns and the expression of conductances in adult stomatogastric ganglion (STG) neurons as a function of time in primary cell culture. When first plated in culture, these neurons had few active properties. After $1 \mathrm{~d}$ in culture they produced small action potentials that rapidly inactivated during maintained depolarization. After $2 \mathrm{~d}$ in culture they fired large action potentials tonically when depolarized, and their properties resembled very closely the properties of STG neurons pharmacologically isolated in the ganglion. After 3- $4 \mathrm{~d}$ in culture, however, their electrical properties changed and they fired in bursts when depolarized. We characterized the currents expressed by these neurons in culture. They included two TTX-sensitive sodium currents, a calcium current, a delayed-rectifier-like current, a calcium-dependent potassium current, and two A-type currents. The changes in firing properties with time in culture were accompanied by an increase in inward and decrease in outward current densities. A single-compartment conductance-based model of an STG neuron was constructed by fitting the currents measured in the biological neurons. When the current densities in the model neuron were matched to those measured for the biological neurons in each activity state, the model neuron closely reproduced each state, indicating that the changes in current densities are sufficient to account for the changes in intrinsic properties. These data indicate that STG neurons isolated in culture change their intrinsic electrical properties by selectively adjusting the magnitudes of their ionic conductances.

Key words: stomatogastric ganglion neurons, culture, expression of conductances, bursting, voltage clamp, neuronal modelling

The electrical properties of neurons are produced by a large array of voltage- and time-dependent ionic conductances (Llinás, 1988; Hille, 1992). There is constant turnover in the membrane of the channels underlying these conductances, and during development neurons may change size and shape and add membrane to neuronal compartments requiring a particular ratio of

\footnotetext{
Received Oct. 6, 1994; revised Nov. 30, 1994; accepted Dec. 5, 1994

We thank Zheng Liu for verifying the model STG neuron in another modeling environment, and L. F. Abbott for many helpful discussions and a critical reading of the manuscript. This work was supported by the Human Frontiers Science Program, a McKnight Foundation Development Award (E.M.), and the W. M. Keck Foundation.

Correspondence should be addressed to Gina Iurrigiano, Department of Biology, Brandeis University, Waltham, MA 02254.

"Present address: Laboratoire de Neurobiologie et Physiologie Comparées, CNRS, 33120 Arcachon, France.

Copyright $(\mathcal{C} 1995$ Society for Neuroscience $0270-6474 / 95 / 153640-13 \$ 05.00 / 0$
}

conductances. Modulation of individual conductances can profoundly alter the clcctrical propertics of neurons (Kaczmarek and Levitan, 1987), and modeling studies have shown that a neuron's intrinsic properties are very sensitive to small changes in the magnitude of conductances (Rinzel and Lee, 1987; Golowasch et al., 1992; Lockery and Spitzer, 1992; Guckenheimer et al., 1993). Channel expression is therefore a dynamic process in which small changes in channel density can produce large changes in neuronal properties. This sensitivity suggests that to achieve and maintain appropriate electrical activity, neurons must continually and selectively adjust the magnitudes of their conductances. To address this issue, we study the expression of conductances in stomatogastric ganglion (STG) neurons of the spiny lobster as they acquire active properties after plating in primary cell culture.

The STG is composed of approximately 30 neurons that participate in two rhythmic motor programs. Most STG neurons are conditional bursters that fire tonically when pharmacologically isolated in the ganglion, but can be induced to burst under the influence of neuromodulatory substances (Harris-Warrick et al., 1992). When STG neuronal somata are dissociated and placed into culture they have large outward and only small inward conductances, because inward currents are not expressed in the soma to a significant degree (Graubard and Hartline, 1991; Golowasch and Marder, 1992b). To regain active properties, they must generate and insert the channels for inward conductances, and balance these with the existing large outward conductances. After $2 \mathrm{~d}$ completely isolated in culture STG neurons fire tonically as they do when pharmacologically isolated in the ganglion. After 3-4 d, however, the majority of STG neurons change their intrinsic properties and become endogenous bursters (Panchin et al., 1993; Turrigiano and Marder, 1993; Turrigiano et al., 1994).

This finding raises several interesting questions. First, what changes in conductances underlie the modification in intrinsic properties? Second, how do these conductances interact to produce the different activity patterns observed? Third, are STG neurons selectively adjusting the magnitudes of their conductances in culture, or are the changes in activity a simple consequence of continued addition of a particular ratio of conductances?

To address these questions, we characterized in more detail the properties of STG neurons isolated in culture. We found that they move through three distinct activity states: after $1 \mathrm{~d}$ in culture, most neurons produce inactivating spikes, after $2 \mathrm{~d}$ most fire tonically, and after 3 and $4 \mathrm{~d}$, most fire in bursts. We characterized the conductances expressed in culture, and asked how the inward and outward current densities changed with time in 
culture. We found a proportional increase in inward and decrease in outward current densities, indicating selective regulation of the balance of conductances in culture. Finally, we constructed a single-compartment, conductance-based model of an STG neuron that incorporated the conductances measured in culture. This model shows that the changes in current densities measured are sufficient to account for the changes in intrinsic neuronal properties.

\section{Materials and Methods}

Animals. Specimens of the California spiny lobster, Panulirus interruptus, were obtained from Marinus (Long Beach, CA) and maintained in an artificial seawater aquarium at $15^{\circ} \mathrm{C}$ without food until used.

Composition of salines and media. $P$. interruptus stock solution contained (in $\mathrm{mM}$ ) $\mathrm{NaCl}, 821.1 ; \mathrm{KCl}, 20.24 ; \mathrm{CaCl}_{2} \cdot \mathrm{H}_{2} \mathrm{O}$, 26.34; $\mathrm{MgSO}_{4}, 18.32 ; \mathrm{Na}_{2} \mathrm{SO}_{4}, 7.8$; HEPES, 10. P. interruptus saline contained (in $\mathrm{mM}$ ) $\mathrm{NaCl}, 478 ; \mathrm{KCl}, 12.9 ; \mathrm{CaCl}_{2} \cdot \mathrm{H}_{2} \mathrm{O}, 13.7$; $\mathrm{MgSO}_{4}, 10 ; \mathrm{Na}_{2} \mathrm{SO}_{4}, 3.9$; Tris base, 6; maleic acid, 5.1.

Isolation of neurons. Neurons were isolated and plated into primary cell culture using a modification of previous techniques (Turrigiano and Marder, 1993). Animals were anesthetized by cooling on ice. Ganglia were removed from the animal, transferred to a sterile Sylgard-lined petri dish in a laninar flow hood, and pinned out in defined medium (composition: sterile Leibowitz-15 diluted 1:1 with sterile-filtered $P$. interruptus stock solution, final $\mathrm{pH} 7.5-7.6$ ). Ganglia were incubated in sterile saline containing $2 \mathrm{mg} / \mathrm{mL}$ subtilisin (Sigma, type VII) for $1.5 \mathrm{hr}$ at $20^{\circ} \mathrm{C}$, then washed for $1 \mathrm{hr}$ with defined medium. Individual somata with a length of primary neurite were removed from the ganglion by aspiration into fire-polished micropipettes with a tip diameter of 100-200 $\mu \mathrm{m}$. They were then plated onto individual uncoated plastic $35 \mathrm{~mm}$ culture dishes (Nunclon) in defined me dium containing $25 \mathrm{U} / \mathrm{ml}$ each penicillin and streptomycin. Cultures were incubated at $20^{\circ} \mathrm{C}$.

Physiological recordings. Culture dishes were transferred to the recording setup and examined and photographed using a Nikon Diphot inverted microscope with modulation contrast optics. Two-electrode voltage-clamp (TEVC) and current-clamp, or single-electrode discontinuous current-clamp (DCC) recordings were obtained using $\mathrm{K}_{2} \mathrm{SO}_{4}$-filled microelectrodes $\left(0.6 \mathrm{M} \mathrm{K}_{2} \mathrm{SO}_{4}\right.$ $+20 \mathrm{mM} \mathrm{KCl} ; 8-12 \mathrm{M} \Omega$ resistances), and an Axoclamp-2A amplifier, at room temperature $\left(22--25^{\circ} \mathrm{C}\right)$. For DCC recordings, switching frequencies of $4-5 \mathrm{kHz}$ were used and the clamp output was continuously monitored. For TEVC experiments, electrodes were coated with Sylgard up to approximately $20 \mu \mathrm{m}$ from the tip to reduce capacitance. The voltage clamp was driven and the voltage and current data recorded using a $33 \mathrm{MHz}$ 386 PC-compatible computer and the pCLAMP software package (Axon Instruments, CA), and data were analyzed using either pCLAMP or AXOGRAPH (Axon Instruments, CA). The preparation was grounded using a silver/silver chloride ground connected to the bath by an agar bridge (4\% agar in $0.6 \mathrm{M} \mathrm{K}_{2} \mathrm{SO}_{4}+20 \mathrm{mM}$ $\mathrm{KCl}$ ). For pharmacological manipulations, agents were added at up to $20 \mathrm{~mm}$ concentrations without ionic compensation; for higher concentrations ions were removed from the physiological saline to compensate. The capacitance was determined by stepping neurons from -80 to $-60 \mathrm{mV}$ for $5 \mathrm{msec}$ (by which time the clamp had settled) and integrating the resulting capacitative transient.

Only neurons with resting membrane potentials $\left(V_{m}\right)$ between -40 and $-70 \mathrm{mV}$, resting input resistences $\left(R_{\mathrm{in}}\right)$ above $20 \mathrm{M} \Omega$ and no morphological signs of damage were used. For the voltage-clamp studies, electrotonically compact neurons isolated with short primary neurites were used; no differences in activity were noted between these neurons and neurons with more extensive growth. Following each experiment the electrode was withdrawn from the neuron and the offset measured. Voltageclamp experiments with offsets $>2 \mathrm{mV}$ were discarded. Data are expressed as mean \pm standard error of the mean (SEM) for the number of experiments indicated.

Neural simulations. A model STG neuron was constructed and simulations run on a Macintosh IIfx computer using the MAXIM software package. MAXIM is general-purpose software for simulating single neurons and small networks (LeMasson, unpublished observations). Experimental data describing the voltage and time dependence of activation and inactivation for each conductance were graphically or numerically entered. Full activation and inactivation curves were obtained with either a polynomial fitting routine (using Newton's algorithm) or a general fitting method (least-squares fit) to extract the parameters of the equations given in Table 1. In both cases correlation coefficients were $>0.98$. Both procedures gave identical results.

For each current, simulated voltage-clamp experiments were run, and the resulting family of current traces fit by eye to a representative fanily of current traces from the biological neurons, by adjusting parameters of the model current. Once each current was fit the activation and inactivation parameters were fixed and were not varied further. To determine if the model neuron could reproduce the current-clamp behavior of the biological neurons, all parameters of the model neuron remained fixed as described except for the maximal conductance $(\bar{g})$ values for the voltage-dependent conductances. The $g$ values were adjusted in simulated voltage- clamp experiments until the peak currents at a given potential matched the mean peak current value for the biological neurons. As current density measurements were not obtained for $I_{h}, \bar{g}_{k}$ was fixed at a value that gave a sag potential during hyperpolarizing pulses within the physiological range in simulated current-clamp experiments, and was not varied further.

Nine voltage- and time-dependent conductances were used to fit the experimental voltage-clamp data. The fast sodium current $\left(I_{\mathrm{Na}}\right)$ and the persistent sodium current $\left(I_{\mathrm{Nap}}\right)$ were modeled as two separate conductances. To fit both the inactivating and persistent components of the calcium current, two model currents with similar activation parameters were needed, one with inactivation $\left(I_{\mathrm{Ca}}\right)$, and one with no inactivation $\left(I_{\mathrm{Ca} 2}\right)$. The ratio of the maximal conductances ( $\bar{g}$ values) for these two components was adjusted to fit a representative set of current traces and then fixed at this ratio $\left(6 \bar{g}_{\mathrm{Ca}}: 1 \bar{g}_{\mathrm{Ca}}\right)$. This ratio was kept constant in model experiments in which $\bar{g}$ values were varied to match the size of the model currents to the values measured in the biological neurons. The transient outward currents were fit with two components with similar activation and inactivation curves, but with different time constants of inactivation, one fast $\left(I_{A}\right)$ and one slow $\left(I_{\mathrm{As}}\right)$. These two components were fit in the same manner as the calcium currents, and the ratio fixed at $2.8 \bar{g}_{\mathrm{A}}: 1 \bar{g}_{\mathrm{As}}$. The calcium- dependent potassium current $\left(I_{\mathrm{KCa}}\right)$ was modeled with both voltage- and calcium-dependent activation, as previously described (Buchholtz et al., 1992). We could adequately account for inactivation of $I_{\mathrm{KCa}}$ by inactivation of $I_{\mathrm{Ca}}$, and so $I_{\mathrm{KCa}}$ was modeled without inactivation. The TEA-sensitive outward current was fit by adjusting the $\bar{g}$ values of the delayed-rectifier potassium current $\left(I_{\mathrm{K} d}\right)$ and $I_{\mathrm{KCa}}$ to fit a representative set of cur- 
Table 1. Equations describing the activation and inactivation properties of the ionic currents of the model STG neuron

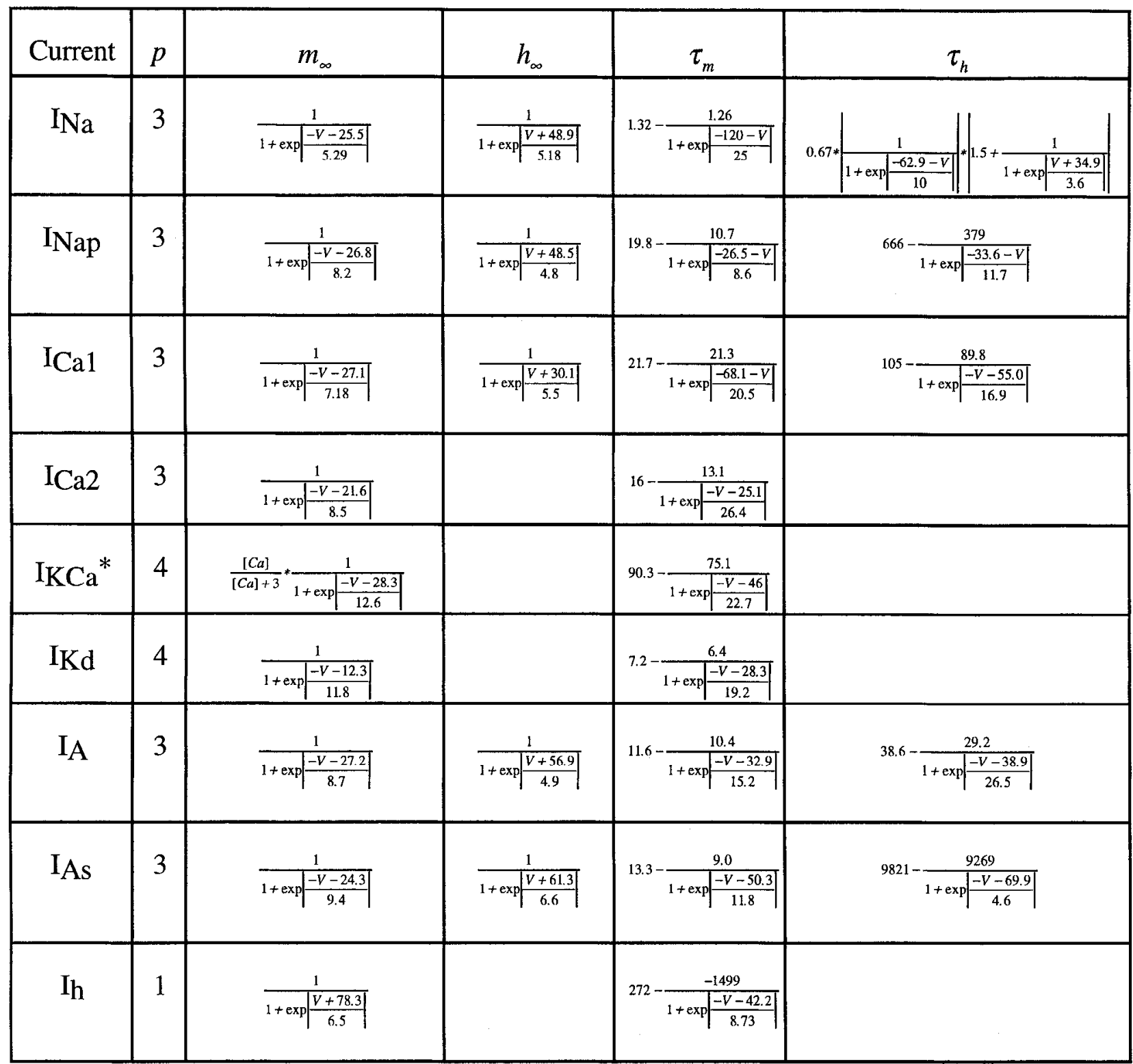

rent traces and fixing the ratio of the $\bar{g}$ s at these values $\left(1 \bar{g}_{\mathrm{K} d}\right.$ : $\left.4 \bar{g}_{\mathrm{KCa}}\right) . I_{h}$ was not directly fit to voltage- clamp data, but was modified from previous descriptions (Golowasch and Marder, $1992 \mathrm{~b}$ ) to reproduce the current-clamp behavior of cultured neurons when hyperpolarized.

Equations. These nine voltage- and time-dependent conductances and leak conductance $\left(I_{t}\right)$ were used to fit the experimental data, and so

$$
\begin{array}{r}
C \frac{d V}{d t}=-\left(I_{\mathrm{Na}}+I_{\mathrm{Na} p}+I_{\mathrm{Ca}_{1}}+I_{\mathrm{Ca}_{2}}+I_{\mathrm{K} d}\right. \\
\left.+I_{\mathrm{KCa}}+I_{\mathrm{A}}+I_{\mathrm{A} s}+I_{h}+I_{l}\right),
\end{array}
$$

where $C=$ membrane capacitance. The voltage- and time-dependent currents were described using modified Hodgkin-Huxley (1952) equations, where each current is a product of a maximal conductance, activation, and inactivation terms, and the driving force for the ion(s) carrying the current:

$$
I=\bar{g} m^{p} h^{q}\left(V-E_{r}\right),
$$

where $\bar{g}$ is the maximal conductance, $m$ and $h$ are activation and inactivation terms that vary between 0 and 1 , and $E_{r}$ is the equilibrium potential for the ion carrying the current. For each current, activation and inactivation were considered to be first order processes, where

$$
\tau_{m}(V) \frac{d m}{d t}=m_{\infty}(V)-m
$$

and

$$
\tau_{h}(V) \frac{d h}{d t}=h_{x}(V)-h
$$

The steady-state activation $\left(m_{\infty}\right)$ and inactivation $\left(h_{\infty}\right)$ equations, and the values for $p$ (which governs the steepness of activation) for each current are given in Table 1. The value for $q$ (which 
governs the steepness of inactivation) was 1 for every inactivating model current. Equations were integrated using the Runge Kutta scheme (Koch and Segev, 1989), which gave good stability and accuracy compared to other classical integration schemes.

The intracellular calcium concentration was considered to be a function of calcium influx via the calcium currents, and removal of calcium through buffering, which was modeled as a simple exponential decay. The equation describing the intracellular calcium dynamics was

$$
\frac{d[\mathrm{Ca}]}{d t}=-A * I_{\mathrm{Ca}}-B *[\mathrm{Ca}]+C,
$$

where $A$ is the increase in $[\mathrm{Ca}] / \mu \mathrm{A}$ of calcium current $(100$ $\mu \mathrm{M} / \mathrm{nC}), B$ is the time constant of calcium removal $(5 \mathrm{msec})$, and $C$ is the resting calcium concentration (50 nM).

The capacitance was set to $0.33 \mathrm{nF}$ and the leak conductance was set to $1.4 \times 10^{-5} \mathrm{mS}$ to give a model neuron input resistance of $70 \mathrm{M} \Omega$ and a resting potential of $-56 \mathrm{mV}$. Both these values are within the standard error of the average values for the biological neurons in culture (Turrigiano et al., 1994). The reversal potentials were $E_{\mathrm{Na}}=50 \mathrm{mV}, E_{\mathrm{Ca}}=150 \mathrm{mV}, E_{\mathrm{K}}=-80 \mathrm{mV}$, $E_{h}=-20 \mathrm{mV}$, and $E_{l}=-65 \mathrm{mV}$ (as for a mixed $\mathrm{Cl}^{-} / \mathrm{K}^{+}$ conductance).

\section{Results}

Change in the properties of STG neurons with time in culture To characterize the changes in electrical properties of STG neurons with time in culture, neuronal activity was recorded $1-4 \mathrm{~d}$ after plating. To classify the activity patterns of neurons, a series of hyperpolarizing and depolarizing current steps were delivered to each neuron and the activity recorded. Neurons were classified as producing inactivating spikes if the action potentials underwent a damped decay in amplitude during a prolonged depolarization (Fig. $1 A$ ). Neurons were classified as tonic if they continued to fire large action potentials during a prolonged depolarization (Fig. 1B). Neurons that showed spike accommodation but little or no decay in action potential amplitude were also classified as tonic. Neurons were classified as bursting if their membrane potentials underwent slow oscillations that were greater than $5 \mathrm{mV}$ in amplitude from peak to trough, either spontaneously or when depolarized (Fig. 1C). Approximately $10 \%$ of neurons that fired tonically at the beginning of a depolarizing current pulse began to fire in bursts after several seconds of depolarization. These neurons were classified as tonic if the transition to bursting behavior required (1) very strong depolarization or (2) more than $5 \mathrm{sec}$ of depolarization; otherwise these neurons were classified as bursting. Approximately $5 \%$ of the neurons had no active properties and were excluded from this analysis.

In a few cases, individual neurons were recorded from on successive days and returned to maintenance medium between recording sessions. Such a recording is shown in Figure $2 A$. After $1 \mathrm{~d}$ in culture, this neuron produced inactivating spikes when depolarized. After $2 \mathrm{~d}$ in culture, the neuron fired action potentials tonically, and after $3 \mathrm{~d}$ in culture, the neuron fired in bursts when depolarized. A similar trend was seen in the pooled data obtained from neurons recorded from on days $1,2,3$, or 4 (Fig. $2 B$ ). On day $1,76 \%$ of the neurons $(n=50)$ produced inactivating spikes; on day $2,62 \%(n=53)$ fired tonically, and on days 3 and $4,61 \%(n=87)$ and $67 \%(n=19)$, respectively, fired in bursts. The resting potentials and input resistances did

\section{A. Inactivating Spikes}

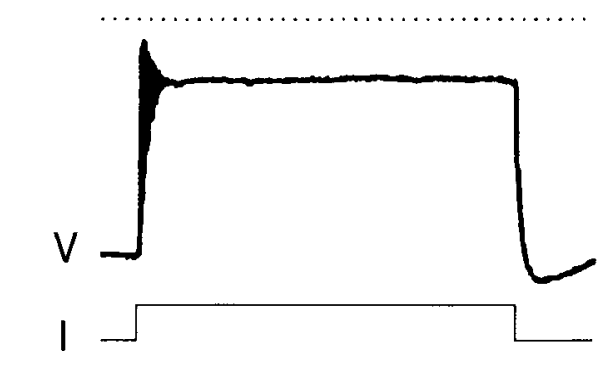

\section{B. Tonic}

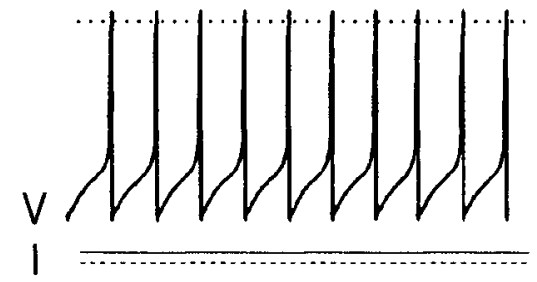

\section{Bursting}

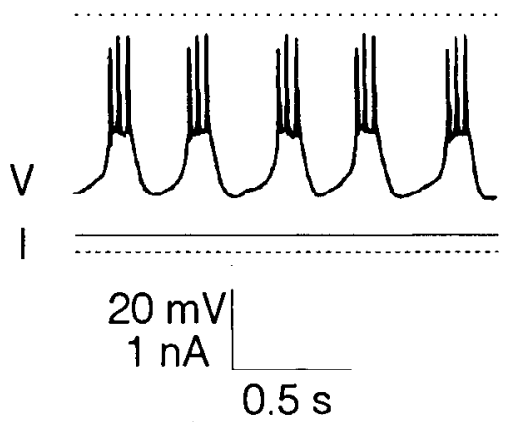

Figure 1. Three lypes of neurond activity recorded from STG neurons in culture. $A$, Inactivating spikes. When depolarized, the neuron fires small-amplitude action potentials that rapidly inactivate. The individual action potentials are clustered at the beginning of the depolarization and cannot be resolved on this time scale. $B$, Tonic. Neuron fires large action potentials tonically when depolarized. $C$, Bursting. The membrane potential undergoes slow-wave oscillations with action potentials riding on top of the slow wave. $V$, intracellular voltage; $I$, current injected through the microelectrode. Dashed line, 0 current; dotted line, $0 \mathrm{mV}$.

not change significantly with time in culture (Turrigiano et al., 1994), and were $-57 \pm 2 \mathrm{mV}$ and $60 \pm 11 \mathrm{M} \Omega$ respectively.

\section{Ionic currents of cultured STG neurons}

Inward currents. Ion substitution experiments have suggested the presence of several inward currents in STG neurons, including TTX-sensitive sodium currents and calcium currents (Gola and Selverston, 1981; Harris-Warrick and Flamm, 1987), but these currents have been difficult to characterize in voltage clamp (Golowasch and Marder, 1992b). Here we describe three inward currents expressed by STG neurons in culture.

Sodium currents. With potassium and calcium currents blocked by $\mathrm{TEA}, \mathrm{Mn}^{2+}$, and a holding potential of $-40 \mathrm{mV}$, a large-amplitude, rapidly activating and inactivating current, $I_{\mathrm{Na}}$, was isolated (Fig. 3A). This current activated above $-40 \mathrm{mV}$, peaked at $-10 \mathrm{mV}$, and had an extrapolated reversal potential 
A

Day 1

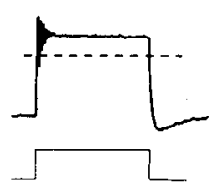

B

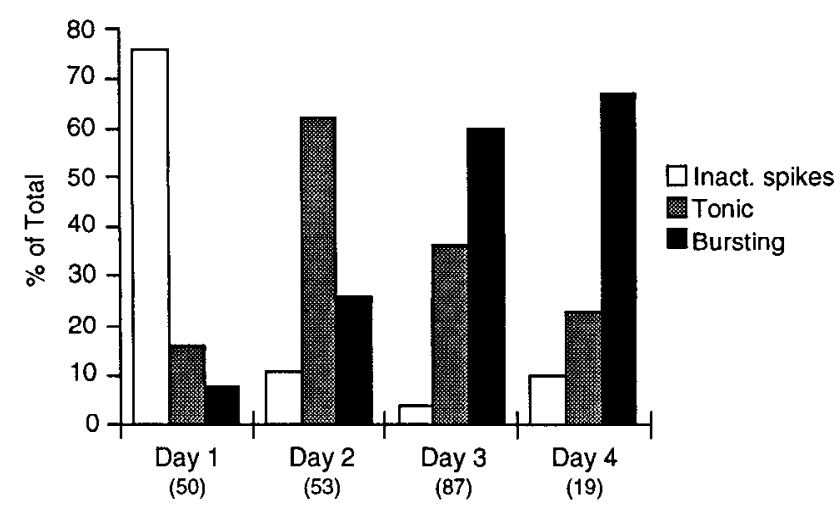

Figure 2. Changes in the activity of STG neurons with time in culture. $A$, Recording from a single neuron after 1,2 , and $3 \mathrm{~d}$ in culture. On each day, the neuron was impaled with a microelectrode, the activity recorded for approximately $1 \mathrm{hr}$, the electrode withdrawn, and the neuron returned to maintenance medium after an extensive wash with sterile saline. Dashed line, $-40 \mathrm{mV}$. B, Percentage of neurons in each activity state after $1-4 \mathrm{~d}$ in culture. of $+55 \mathrm{mV}$ (Fig. $3 B$ ). $I_{\mathrm{Na}}$ activated rapidly, within the first 2 msec following a change in voltage. We were able to achieve settling times for the voltage clamp of within 1 msec, much better than those achieved previously (Golowasch and Marder, 1992b), but not fast enough to determine accurately the activation rates or peak current amplitudes for $I_{\mathrm{Na}}$. The values reported below for the peak current amplitudes are therefore likely to be an underestimate of the actual values. The dependence of $I_{\mathrm{N} a}$ on extracellular sodium was not tested. This current and the current described below were considered to be sodium currents because they were blocked by TTX and reversed around $+50 \mathrm{mV}$.

An additional small-amplitude, persistent TTX-sensitive inward current could be isolated under these conditions. Neurons were given 4-sec-long voltage steps to various potentials from a holding potential of $-50 \mathrm{mV}$, in the presence and absence of TTX. The TTX-sensitive persistent sodium current $\left(I_{\text {Nap }}\right)$ was isolated by subtracting currents elicited in the presence of TTX from those elicited in its absence (Fig. $3 C$ ). $I_{\mathrm{Nap}}$ activated more slowly than $I_{\mathrm{Na}}$, peaking within about $15 \mathrm{msec}$, and inactivated very slowly. $I_{\mathrm{Nap}}$ activated at a lower threshold than $I_{\mathrm{Na}}$, beginning to activate at $-50 \mathrm{mV}$, peaked at $-10 \mathrm{mV}$, and had an extrapolated reversal potential of $+48 \mathrm{mV}$ (Fig. $3 D$ ).

Calcium currents. With other voltage-dependent currents blocked by $20 \mathrm{~mm}$ TEA, $0.1 \mu \mathrm{M}$ TTX, and a holding potential of $-50 \mathrm{mV}$, a net inward current could be elicited with depolarizing voltage steps. This current was blocked by $10 \mathrm{~mm} \mathrm{Mn^{2+ }}$ This $\mathrm{Mn}^{2+}$-sensitive inward current $\left(I_{\mathrm{Ca}}\right)$ was carried by calcium, as changing the extracellular calcium concentration shifted the magnitude and extrapolated reversal potential of the current (data not shown). $I_{\mathrm{Ca}}$ was easier to isolate with $\mathrm{Ba}^{2+}$ substituted
Figure 3. TTX-sensitive currents. $A$, $I_{\mathrm{Na}}$. A voltage step from -40 to -10 $\mathrm{mV}$ in the presence of $20 \mathrm{~mm}$ TEA elicited a large rapidly inactivating current that was blocked by $0.1 \mu \mathrm{M}$ TTX. $I$, current; $V$, voltage. $B$, Current volt age plot of $I_{\mathrm{Na}}$, isolated by subtracting currents elicited in the presence of TTX from currents elicited in the absence of TTX. For each experiment, peak current at each voltage was normalized to the peak current for the experiment. Each point is the mean \pm SEM for 14 experiments. $C, I_{\mathrm{N} x y y}$. Subtracting currents elicited by long voltage steps in the presence of TTX from those elicited in the absence of TTX isolated a small, persistent inward current. $I$, current; $V$, voltage command. $I_{\mathrm{Na}}$ cannot be distinguished in this recording be cause a low sampling rate was used for these long voltage commands. $D$, Current- voltage plot of $I_{\mathrm{Na}, \mathrm{r}}$. For each experiment, peak current at each voltage was normalized to the peak current for the experiment. Each point is the mean \pm SEM for 10 experiments.
A

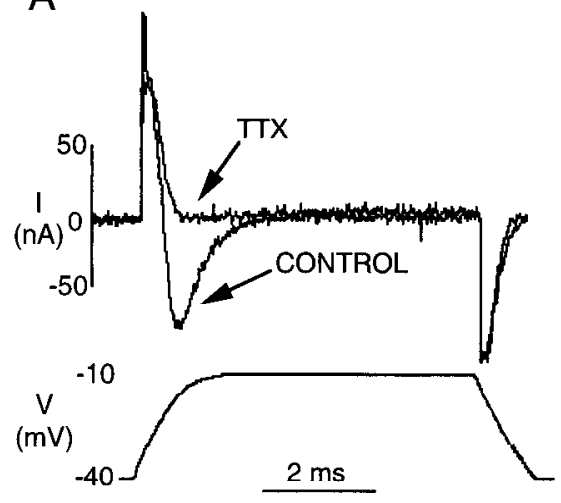

B

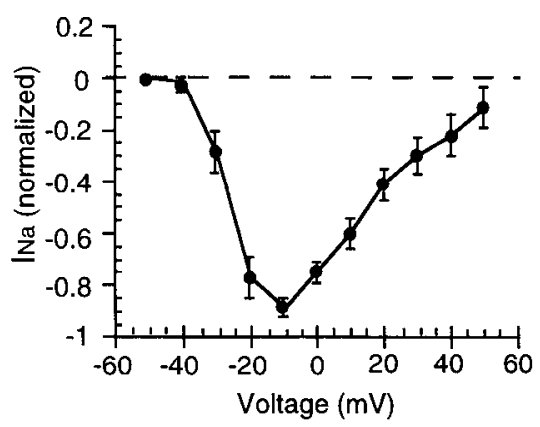

C

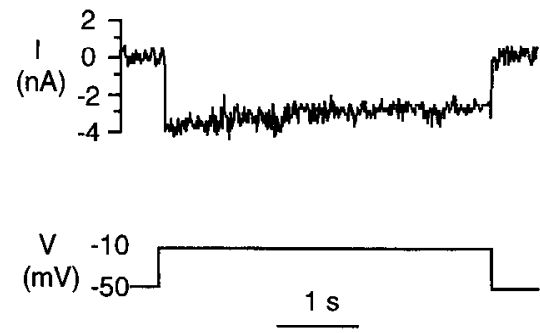

D

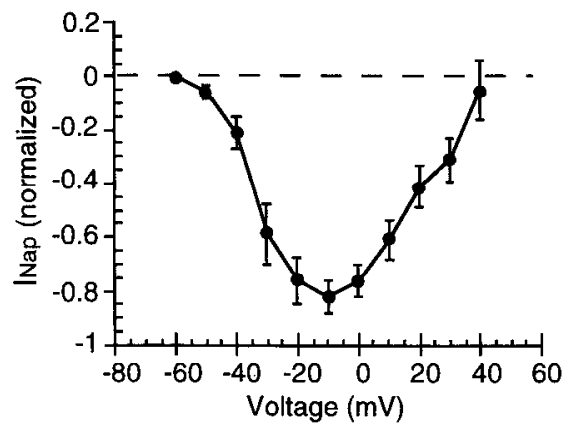


A
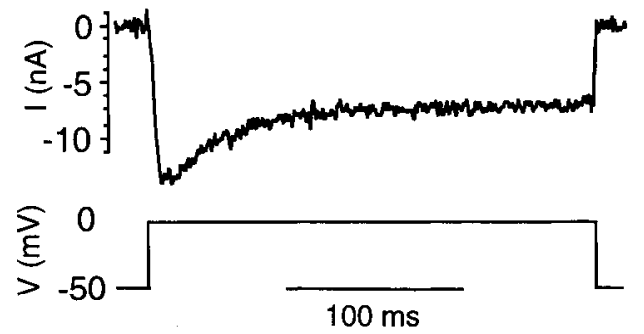

B



Figure 4. Calcium current. $A, I_{\mathrm{Ca}}$. Subtraction of currents elicited in the presence of $10 \mathrm{mM} \mathrm{Mn}^{2+}$ from currents elicited in the absence of $\mathrm{Mn}^{2+}$, with $20 \mathrm{mM}$ TEA, $0.1 \mu \mathrm{M}$ TTX, and $\mathrm{Ba}^{++}$substituted for $\mathrm{Ca}^{2+}$ in the external solution. $I$, current; $V$, voltage command. $B$, Current-voltage plot of $I_{\mathrm{Ca}}$. For each experiment, peak current at each voltage was normalized to the peak current for the experiment. Each point is the mean \pm SEM for 10 experiments.

for $\mathrm{Ca}^{2+}$ in the extracellular solution, as $\mathrm{Ba}^{2+}$ blocked a residual small outward current not blocked by TEA. In some experiments, $\mathrm{Cs}^{+}$was injected intracellularly to block residual $\mathrm{K}^{+}$currents. To characterize the $\mathrm{Mn}^{2+}$-sensitive component of the $\mathrm{Ba}^{2+}$ current, currents elicited in the presence of $\mathrm{Mn}^{2+}$ were subtracted from those elicited in the absence of $\mathrm{Mn}^{2+}$. Measured under these various experimental conditions, $I_{\mathrm{Ca}}$ had both an inactivating and a persistent component (Fig. $4 A$ ). $I_{\mathrm{Ca}}$ activated above $-40 \mathrm{mV}$, and peaked between 0 and $+10 \mathrm{mV}$ (Fig. $4 B$ ). This current was also sensitive to $\mathrm{Cd}^{2+}$ and $\mathrm{Co}^{2+}$ but these agents were difficult to reverse and had toxic effects on cultured neurons at concentrations sufficient to block the current completely. In addition, in a few experiments a low-threshold calcium current was identified that was not sensitive to $\mathrm{Mn}^{2+}$ or to $\mathrm{Ni}^{2+}$, but was blocked by $\mathrm{Cd}^{2+}$. It was not possible to characterize this current fully, as in most experiments it was contaminated by the large $I_{\mathrm{A}}$ elicited with the same voltage-clamp protocols.

Hyperpolarization-activated inward current, $\mathbf{I}_{\mathrm{h}}$. 'I his current has been characterized previously in crab STG neurons (Golowasch and Marder, 1992b; Kiehn and Harris-Warrick, 1992). In cultured Panulirus STG neurons, this current is activated by hyperpolarizing voltage steps below $-60 \mathrm{mV}$, activates slowly, with a time constant of several seconds, and has a reversal potential around $-20 \mathrm{mV}$.

Outward currents. Golowasch and Marder (1992b) characterized the outward currents expressed by the lateral pyloric (LP) neuron of the crab STG. These currents include a delayed-rectifier $\left(I_{\mathrm{K} d}\right)$ and a calcium-dependent potassium current $\left(I_{\mathrm{KCa}}\right)$, both of which are sensitive to TEA, and a transient potassium current $\left(I_{\mathrm{A}}\right)$. Similar outward currents are expressed in cultured STG neurons, as described below.

TEA-sensitive outward currents. To isolate outward currents, neurons were put into $0.1 \mu \mathrm{M}$ TTX to block sodium currents, and given a series of voltage steps from a holding potential of $-50 \mathrm{mV}$. Voltage steps to potentials above $-30 \mathrm{mV}$ elicited a large outward current, $80-90 \%$ of which was blocked by $20 \mathrm{~mm}$ TEA. To isolate the TEA-sensitive component of the outward current, currents elicited in the presence of TEA were subtracted from those elicited in the absence of TEA (Fig. 5A,B). A portion of this outward current was blocked by manipulations that blocked calcium currents (see above), or by replacing $\mathrm{Ca}^{2+}$ with $\mathrm{Ba}^{2+}$ in the saline, which does not activate $I_{\mathrm{KCa}}$. This current thereforc corresponds to the previously described $I_{\mathrm{KCa}}$. The remainder of the TEA-sensitive current showed a delay to onset and no inactivation, indicating that it corresponds to the previously described $I_{\mathrm{K} d}$ (Golowasch and Marder, 1992b).

Transient potassium currents. To isolate the transient potassium currents, neurons were placed into $0.1 \mu \mathrm{M}$ TTX and 20 $\mathrm{mm}$ TEA, and in some cases $10 \mathrm{~mm} \mathrm{Mn}^{2+}$ was also used to block calcium currents. The transient outward currents are inactivated at holding potentials above $-50 \mathrm{mV}$, so to isolate them currents elicited from holding potentials of $-40 \mathrm{mV}$ were subtracted from currents elicited from holding potentials of -80 $\mathrm{mV}$, a potential at which inactivation is completely removed (Fig. $5 C, D$ ). This current activates above $-50 \mathrm{mV}$, and has both a rapidly inactivating and a more slowly inactivating component, as has previously been described (Graubard and Hartline, 1991; Golowasch and Marder, 1992b; Tierney and Harris-Warrick, 1992).

To characterize better the slowly inactivating component of the transient outward current, long voltage steps were given in the presence of TTX, TEA, and $\mathrm{Mn}^{2+}$ (Fig. 6). In current clamp, constant-amplitude depolarizing current injection under these conditions produced a slow depolarization of the membrane potential, with both a fast and a slow component. In voltage clamp, this corresponded to the inactivation of a large outward current, with both a fast $(\tau=100-200 \mathrm{msec})$ and a slow $(\tau=1-3 \mathrm{sec})$ time constant of inactivation. The fast component corresponds to the previously described $I_{\mathrm{A}}$, and the slow component we designate $I_{\mathrm{A} s}$.

Activation of $I_{\mathrm{A}}$ and $I_{\mathrm{As}}$ was measured by stepping the voltage to various potentials from a holding potential of $-100 \mathrm{mV}$. Inactivation was measured by holding the potential at $-100 \mathrm{mV}$, giving long prepulses of $20 \mathrm{sec}$ to voltages between -100 and $+20 \mathrm{mV}$, and then stepping the voltage to $+40 \mathrm{mV}$ (a potential at which the currents are fully activated). Measurement of the elicited currents at 50,500, and $3000 \mathrm{msec}$ gave similar activation and inactivation curves for both components of $I_{\mathrm{A}}$. Both $I_{\mathrm{A}}$ and $I_{\mathrm{A} s}$ activated above $-40 \mathrm{mV}$, and were completely inactivated by holding potentials of $-40 \mathrm{mV}$ or above. There was little overlap in the activation and inactivation curves (Fig. 6).

\section{Change in current densities with time in culture}

The change in electrical properties of STG neurons with time in culture suggested that the balance of conductances in these neurons was changing. To address this possibility, we measured the density of currents for neurons producing inactivating spikes, tonic spikes, or bursts. We measured $I_{\mathrm{Na}}, I_{\mathrm{Naph}}, I_{\mathrm{Ca}}$, the TEA-sensilive outward current (which is composed of $I_{\mathrm{Kd}}$ and $I_{\mathrm{KCi}}$ ), and the peak $I_{\mathrm{A}}$ current (which is composed of $I_{\mathrm{A}}$ and $I_{\mathrm{A} .}$ ). For each 
A

Figure 5. Outward currents expressed in culture. $A$, TEA-sensitive outward currents elicited by depolarizing voltage steps from a holding potential of $50 \mathrm{mV}$. Currents elicited in the presence of $20 \mathrm{~mm}$ TEA were subtracted from those elicited in the absence of TEA to isolate the TEA-sensitive component of the outward current. $B$, Current-voltage plot of the TEA-sensitive current. Peak current was normalized to the current elicited by steps to -10 $\mathrm{mV}$. Each data point is the mean \pm SEM for 20 experiments. $C, I_{\mathrm{A}}$, isolated by subtracting currents elicited from a holding potential of $-40 \mathrm{mV}$ from those elicited from a holding potential of $-80 \mathrm{mV} . D$, Current-voltage plot of $I_{\mathrm{A}}$. Peak current was normalized to the currents elicited by steps to $0 \mathrm{mV}$. Each data point is the mean \pm SEM for 15 experiments. Upper traces in $A$ and $C$ show currents in $\mathrm{nA}$; lower traces show vollage command in inV. Dashed line in $B$ and $D$ indicates zero current.

B

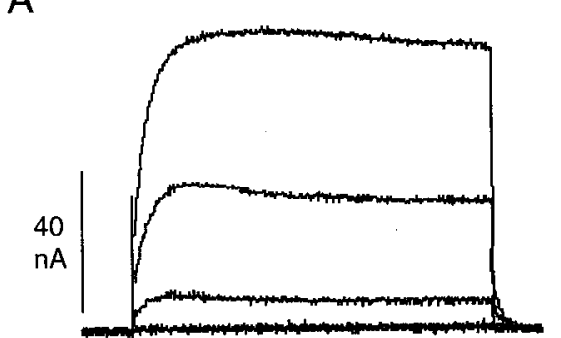

C
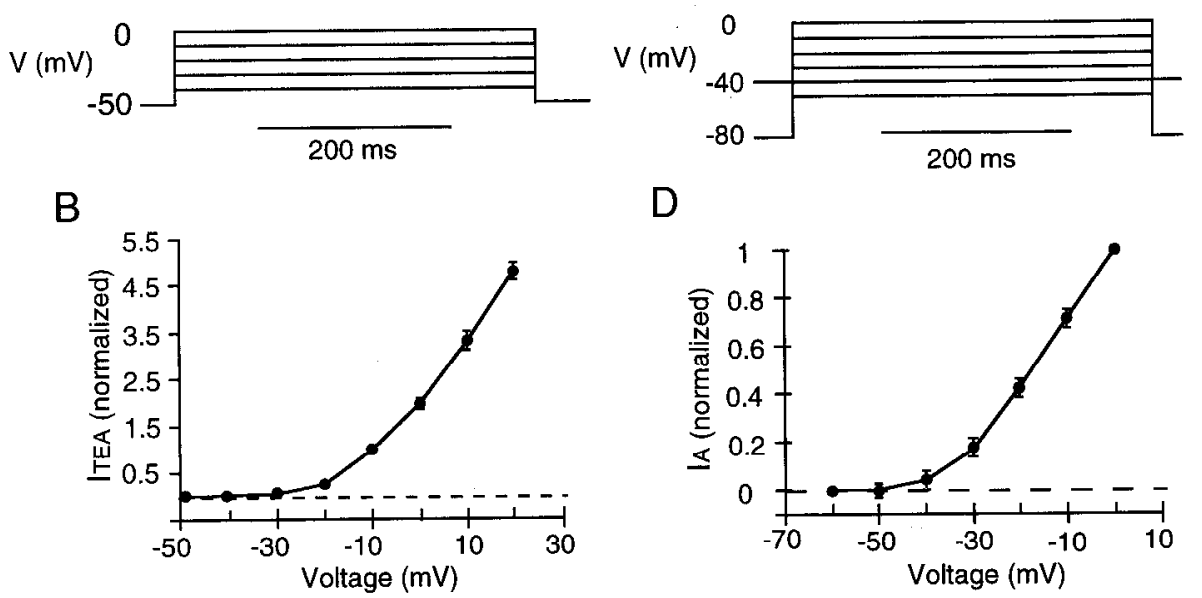

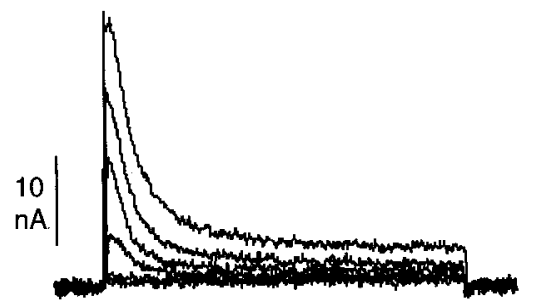

D

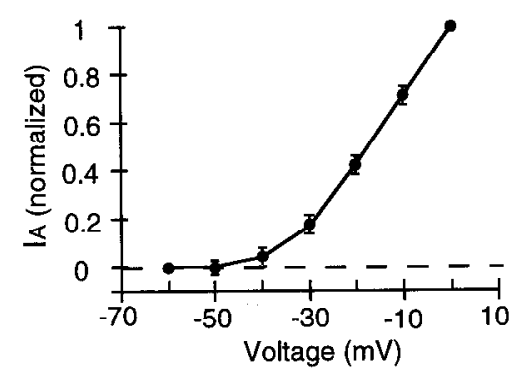

neuron the current was normalized to the capacitance to control for differences in growth and soma size, and therefore is expressed as $\mathrm{nA} / \mathrm{nF}$. For the inward currents, the peak current value was measured, and for the outward currents, the peak current value at $-10 \mathrm{mV}$ was measured, a potential at which the large outward currents could be routinely well clamped. For each point, data from 6-12 neurons were averaged.

As illustrated in Figure 7, the inward current densities increased dramatically as neurons changed from producing inactivating spikes to firing tonically. $I_{\mathrm{Na}}$ increased from $37 \pm 5$ to $74 \pm 12 \mathrm{nA} / \mathrm{nF}, I_{\mathrm{N} a y}$ increased from $3.5 \pm 2.0$ to $12.1 \pm 3.1$ $\mathrm{nA} / \mathrm{nF}$, and $I_{\mathrm{Ca}}$ increased from $6 \pm 2$ to $16 \pm 3 \mathrm{nA} / \mathrm{nF}$. As neurons changed from firing tonically to bursting, there was no further significant change in the sodium current densities. The calcium current, in contrast, increased significantly from $16 \pm$ 3 to $25 \pm 2 \mathrm{nA} / \mathrm{nF}$ as neurons moved from tonic firing to bursting.

While the inward currents increased in density, the opposite pattern was observed for the outward currents. Both the TEAsensitive component of the outward current and $I_{\mathrm{A}}$ decreased dramatically as neurons moved from inactivating spikes to bursting. The TEA-sensitive outward current decreased from $115 \pm$ 21 to $31 \pm 4 \mathrm{nA} / \mathrm{nF}$, while $I_{\mathrm{A}}$ decreased from $111 \pm 11$ to 34 $\pm 8 \mathrm{nA} / \mathrm{nF}$. There was no significant difference in capacitance for ncurons in each state (inactivating spikes, $0.41 \pm 0.06 \mathrm{nF}$; tonic, $0.53 \pm 0.08 \mathrm{nF}$; bursting, $0.47 \pm 0.07 \mathrm{nF}$ ). Capacitance was measured in neurons from which voltage-clamp data were obtained, which were selected for electrotonic compactness. In these neurons, the somatic membrane was large relative to the neuritic membrane, so the majority of the capacitance was contributed by the somata. STG neuronal somata vary in size from approxinately 20 to $100 \mu \mathrm{m}$ in diameter, so the variability in somatic capacitance is likely to be much larger than the change in capacitance due to neurite outgrowth in this population of neurons.
No significant changes in the voltage dependence of activation or inactivation of currents were observed with time in culture. There was sufficient variability in the kinetics of activation and inactivation from neuron to neuron to make it difficult to determine if there were significant changes in kinetics with time in culture.

\section{Neural simulations}

Wc wished to know whether the currents described above are sufficient to account for the observed electrical properties of STG neurons, and whether the measured changes in current density can account for the changes in electrical properties with time in culture. To address these issues, we built a single-compartment model STG neuron in which we incorporated the conductances measured in the biological neurons. Each current in the model neuron was fit to representative biological data using the software package MAXIM (see Materials and Methods). The fits of $I_{\mathrm{Na}}, I_{\mathrm{Ca}}, I_{\mathrm{TEA}}$, and $I_{\mathrm{A}}$ are shown in Figure 8 . Fits were optimized by eye, and deviations from the data were within the variability of the biological data.

The model STG neuron reproduces the biological activity patterns. Once each current in the model was fit to data, the parameters governing voltage and time dependence were fixed. The only parameters varied were the $\bar{g}$ values, which were adjusted so that the densities of each model current matched the average measured current density for the biological neurons. The results are shown in Figure 9. When the current densities were matched in this way, the model neuron reproduced each of the activity patterns observed in the biological neurons. When the current amplitudes matched those measured in neurons producing inactivating spikes, the model neuron also produced inactivating spikes. When the current amplitudes matched those measurcd in tonically firing neurons, the model neuron fired tonically, and the wave form and amplitude of the action potentials in the model were very similar to those of tonically firing biological 


\section{A. Current Clamp}

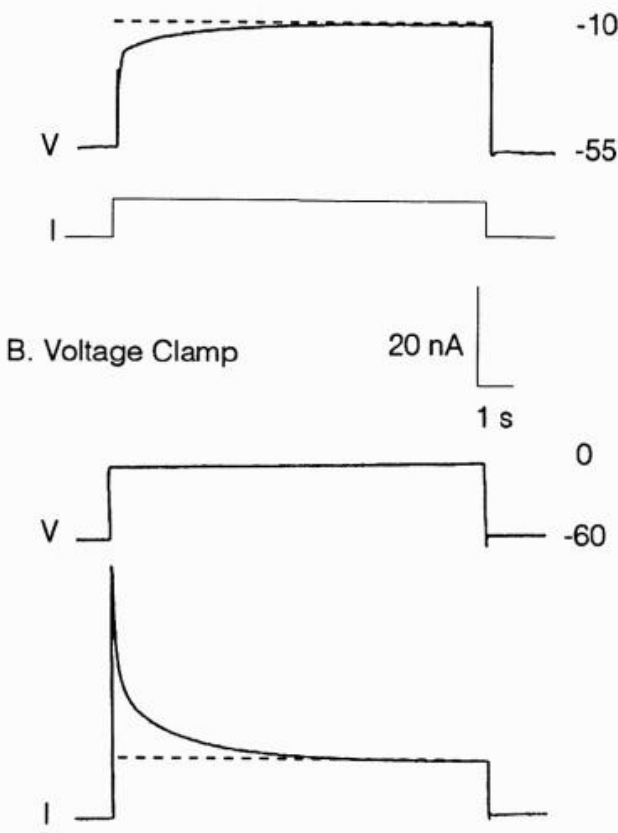

C.

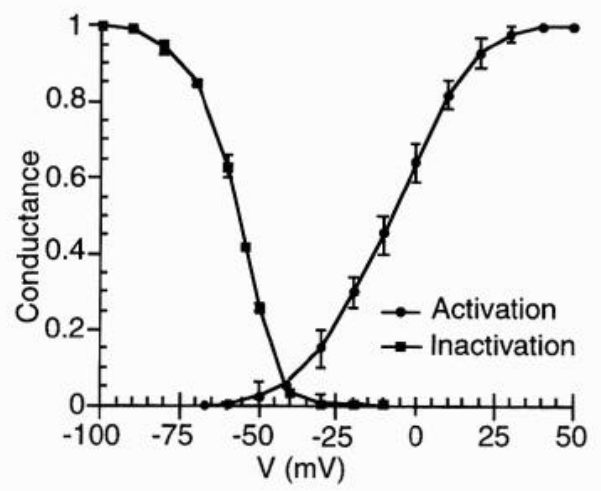

Figure 6. Slowly inactivating outward current, $I_{\mathrm{A}} . A$, Current-clamp recording in the presence of TTX, TEA, and $\mathrm{Mn}^{2+}$. Dashed line indicates $-10 \mathrm{mV} . V$, intracellular voltage; $I$, current injected through the microelectrode. $B$, Voltage clamp of the neuron shown in $A$. Voltage steps from -60 to $0 \mathrm{mV}$ elicited a large outward current that inactivated slowly. Dashed line indicates current remaining after steady state had been reached. Traces were not leak subtracted. $V$, voltage; $I$, current. $C$, Activation and inactivation curves for $I_{\mathrm{A}}$. Conductance $(g)$ was normalized to the maximal conductance, and each point represents the average data from eight experiments.

neurons (the difference in action potential amplitude was due to truncation of the biological spikes by about $10 \mathrm{mV}$ by the mechanical chart recorder). When the currents densities matched those measured in bursting neurons, the model neuron fired in bursts when depolarized, with similar amplitude and wave form of bursts to those of the biological neurons. The model neuron was able to burst over a wide range of frequencies $(2-13 \mathrm{~Hz})$ as injected current was increased, but for current injections above $2 \mathrm{nA}$ the model neuron either fired spikes tonically or the membrane potential "locked up" at depolarized values. The biological neurons behaved similarly, except that the frequency range of bursting was more restricted $(1-5 \mathrm{~Hz})$. A small number of bursting cultured neurons produced much slower bursts, with periods from 5 to $10 \mathrm{sec}$. These neurons may represent neurons
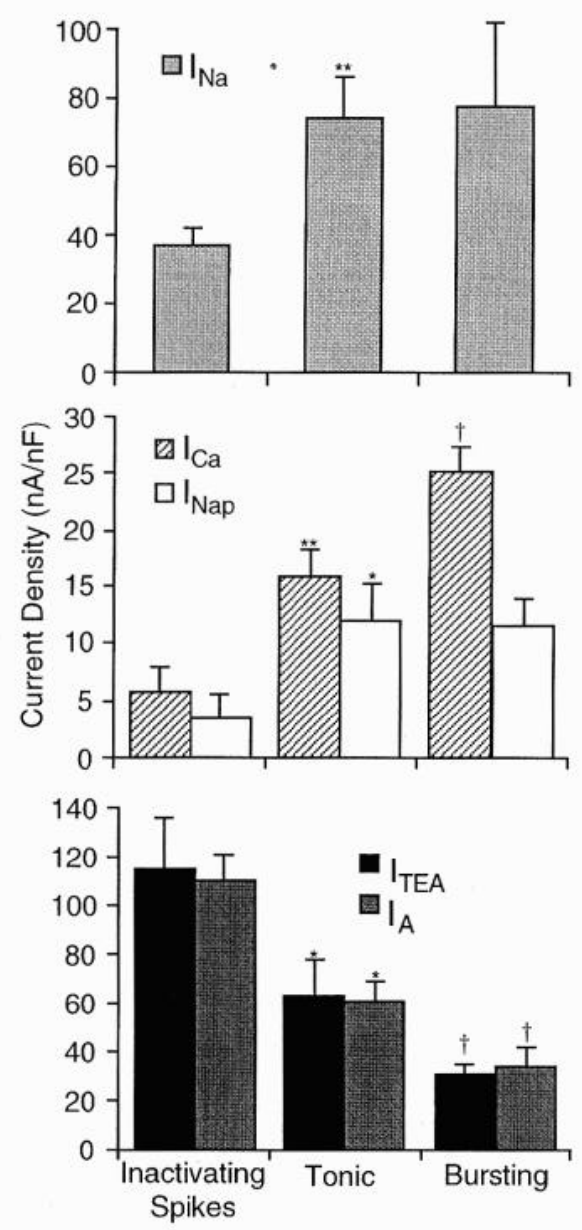

Figure 7. Current densities for neurons in each activity state (Inactivating Spikes, Tonic, and Bursting). For each current measurement the peak current (inward currents) or the peak current elicited by voltage steps to $-10 \mathrm{mV}$ (outward currents) was divided by the capacitance of the neuron and is expressed as $\mathrm{nA} / \mathrm{nF}$. Top, $I_{\mathrm{Na}}$; middle, $I_{\mathrm{Na},}$ and $I_{\mathrm{Ca}}$; bottom, $I_{\text {TEA }}$ and $I_{\mathrm{A}}$. Each histogram represents the average data from 6-12 neurons. *, Tonic significantly different from Inactivating Spikes, unpaired $t$ test, $p<0.05$; **, $p<0.01$. $\dagger$, Bursting significantly different from Tonic, unpaired $t$ test, $p<0.05$.

that participate in the gastric mill rhythm, which has a similar long period. Such slow oscillations could be produced in the model neuron by slowing the kinetics of activation of $I_{\mathrm{Ca}}$ and $I_{\mathrm{KCa}}$ (data not shown).

Changes in activity patterns. To understand better how the ionic currents interact to produce the different activity states, we monitored the current flowing through each conductance during ongoing activity in the model STG neuron (Fig. 10). When the model neuron produces inactivating spikes, $I_{\mathrm{Na}}$ is not large enough to activate $I_{K d}$, and the spikes do not repolarize enough to remove inactivation from $I_{\mathrm{Na}}$; as a consequence, the spikes rapidly inactivate (Fig. 10A). When the model neuron fires tonically, the increase in $I_{\mathrm{Na}}$ strongly activates $I_{\mathrm{K} d}$ during each spike, repolarizing the neuron and removing inactivation from $I_{\mathrm{Na}}$ (Fig. $10 B$ ), so the model neuron fires large action potentials tonically. When the model neuron fires in bursts the increase in $I_{\mathrm{Ca}}$ depolarizes the neuron to produce the depolarizing phase of the burst. The increase in calcium influx strongly activates $I_{\mathrm{KCa}}$, which then repolarizes the burst (Fig. 10C). Burst repolarization is achieved by both inactivation of $I_{\mathrm{Ca}}$ and activation of $I_{\mathrm{KCa}}$, in 
A

Figure 8. Fit of model currents to voltage clamp data. $A, I_{\mathrm{Na}}$. Currents clicitcd by voltage steps from a holding potential of $-50 \mathrm{mV}$ to $-30,-20$, and $-10 \mathrm{mV} . B, I_{\mathrm{Ca}}$. Currents elicited by voltage steps from a holding potential of $-50 \mathrm{mV}$ to $-30,-20,-10$, and 0 $\mathrm{mV} . C, I_{\text {TFA }}$. Currents elicited by voltage steps from a holding potential of $-50 \mathrm{mV}$ to $-30,-20,-10$, and $0 \mathrm{mV}$. $D, I_{\mathrm{A}}$ and $I_{\mathrm{A}, *}$. Currents elicited by voltage steps from a holding potential of $-80 \mathrm{mV}$ to $-30,-20$, and $-10 \mathrm{mV}$. The noisy lines indicate the biological currents, and the smooth lines indicate currents generated by the model neuron in simulated voltage-clamp experiments.

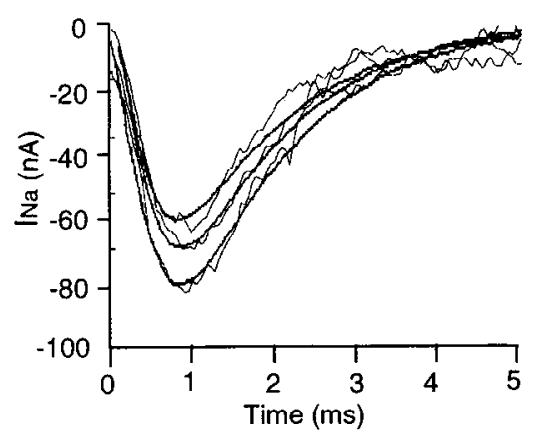

B

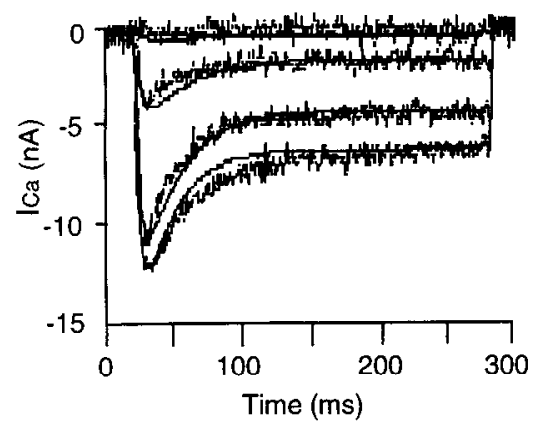

C

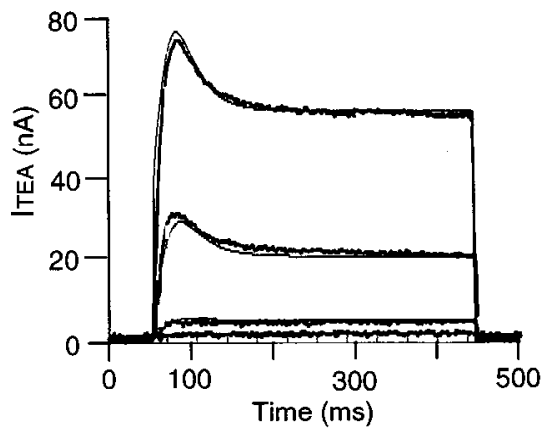

D



contrast to recent models of the bursting neuron $\mathrm{R} 15$ in which inactivation of $I_{\mathrm{Ca}}$ is sufficient to repolarize bursts (Canavier et al., 1991). $I_{\mathrm{Nap}}$ serves to control the level of depolarization, and contributes to the depolarizing ramp leading to each burst, as $I_{\mathrm{Na} p}$ activates in a more hyperpolarized range than $I_{\mathrm{Ca}}$ (Figs. 4, 5 ). In addition, $I_{\mathrm{Na}}$ contributes to the burst by producing a strong depolarization that activates the higher- threshold $I_{\mathrm{Ca}}$. Bursts in the model neuron depend upon both sodium and calcium currents, as has been described for the biological neurons (Gola and Selverston, 1981; Harris-Warrick and Flamm, 1987; Turrigiano and Marder, 1993), and in other bursting model neurons in some parameter regimes (Epstein and Marder, 1990; Canavier et al., 1991).

Activity maps. To understand better the transitions in activity with time in culture, we wished to look at the behavior of the model neuron over a broader range of conductance values than the three points in conductance space examined in Figure 9. To accomplish this, we started with the conductances set as measured for biological neurons producing inactivating spikes, and then systematically increased the tolal inward conductances and decreased the total outward conductances (Fig. 11). Each conductance was varied in $10 \%$ increments between the initial values for neurons producing inactivating spikes, and final values for neurons in a bursting state (see Fig. 7 for these values for each conductance). The $y$-axis shows the $\bar{g}$ values for the inward currents, with 0 being the values for biological neurons producing inactivating spikes, and 1 being the values for biological neurons firing in bursts. The $\mathrm{x}$-axis shows the $\bar{g}$ values for the outward currents, with 1 being the values for biological neurons producing inactivating spikes, and 0 being the values for biological neurons firing in bursts.

When the currents were varied systematically in this way, well-defined regions in conductance space appeared in which the model neuron produced each of the behaviors described (Fig. 11). Some transitional behaviors were observed; for example, on the tonic side of the tonic/bursting boundary the neuron fired spikes in groups, but without a significant slow wave oscillation. A $10 \%$ increase in the inward currents was sufficient to move the neuron from this state (hatched) to a bursting state (crosshatched), indicating that the boundaries between these states are quite sharp. The activity of neurons sitting close to this boundary will therefore be very sensitive to small changes in conductances, as has been noted recently for another model of a bursting STG neuron (Guckenheimer et al., 1993).

In this map inward and outward currents were varied linearly between starting and ending values. While this is reasonably close to the pattern of change observed for most conductances with time in culture, it does not accurately describe the pattern of change of the sodium currents, which increase initially and then stabilize while the calcium currents continue to increase. In other model experiments we have constructed activity maps that reflect this sequence of changes in the inward currents; this results in a more rapid transition from inactivating spikes to tonic firing, but does not otherwise significantly change the shape of the activity space.

The activity map in Figure 11 illustrates that in order for neurons to reach a bursting state, they must first move through a large conductance space in which they fire tonically. In addition, the fastest way to get from inactivating spikes to bursting is to proportionally increase inward currents and decrease outward currents, by following a diagonal line through this conductance space from lower left to upper right. If inward currents are increased and outward currents remain at initial values (a vertical path from lower left to upper left), much bigger changes in the inward currents are required to reach a bursting state. If inward currents remain at initial values and outward currents decrease (a horizontal path from lower left to lower right), a bursting state is never reached.

\section{Discussion}

We have found that, when isolated in primary cell culture, STG neurons change their intrinsic activity from tonic firing to burst- 

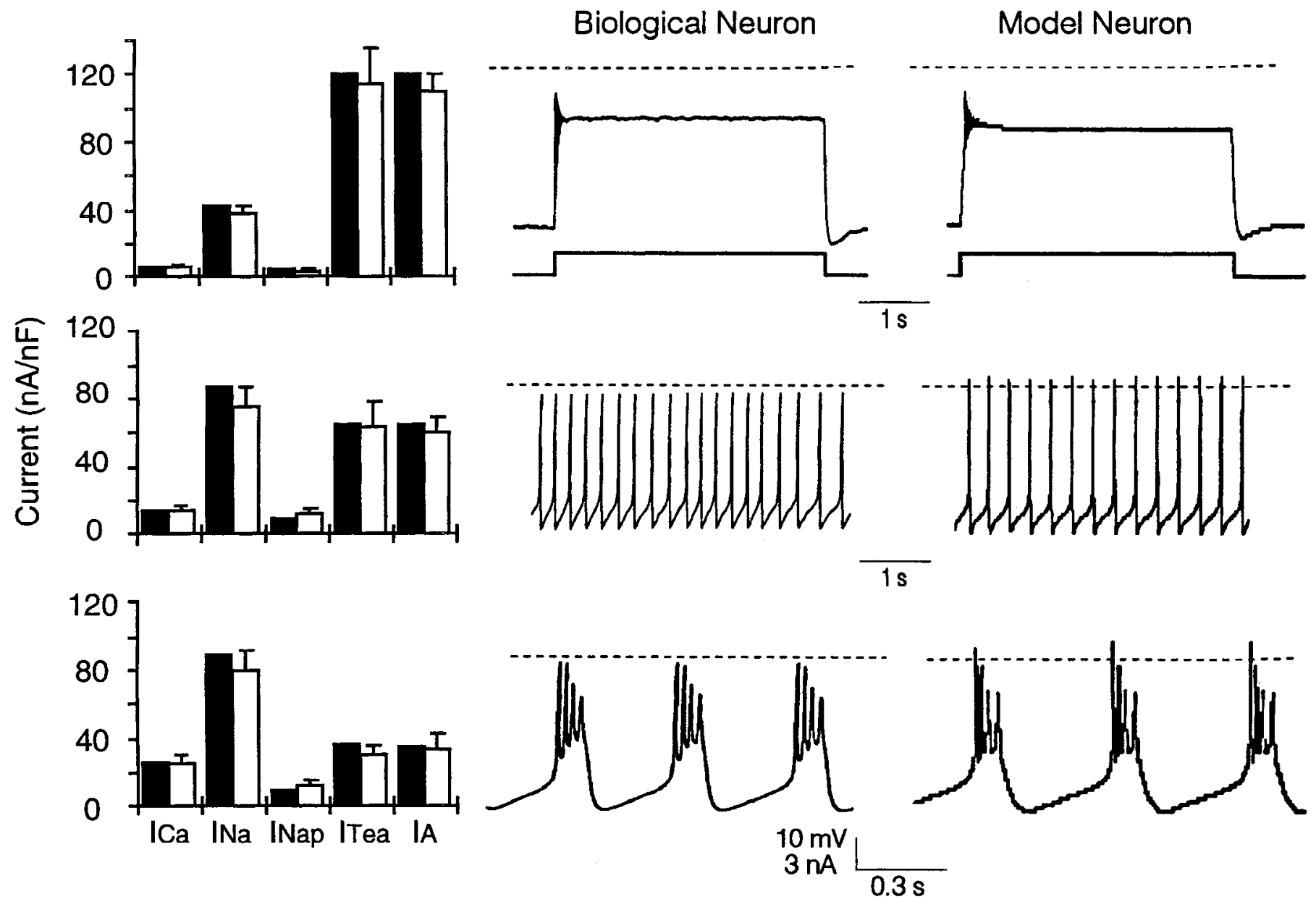

Figure 9. Comparison of the activity of the model and the biological neurons. Histograms represent the mean \pm SEM current density (nA/nF); $\square$, biological currents; $\boldsymbol{\square}$, model currents. The values for the biological neurons are those reported in Figure 7. Peak current densities were measured at $-10 \mathrm{mV}$ for outward currents, and peak values were taken for inward currents. Each panel across shows the activity of the biological and model neurons when the current densities are those indicated by the histograms to the left. Dashed line indicates $0 \mathrm{mV}$ in all panels.
A. Inactivating
C. Bursting

Spikes
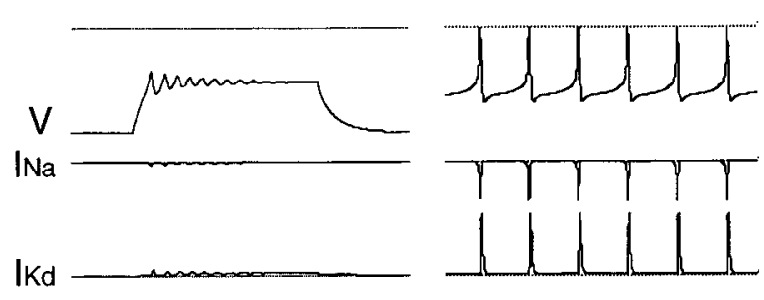

INap

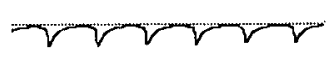

$\mathrm{IKCa}$
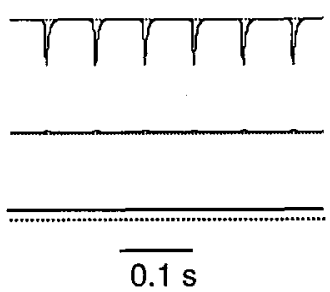
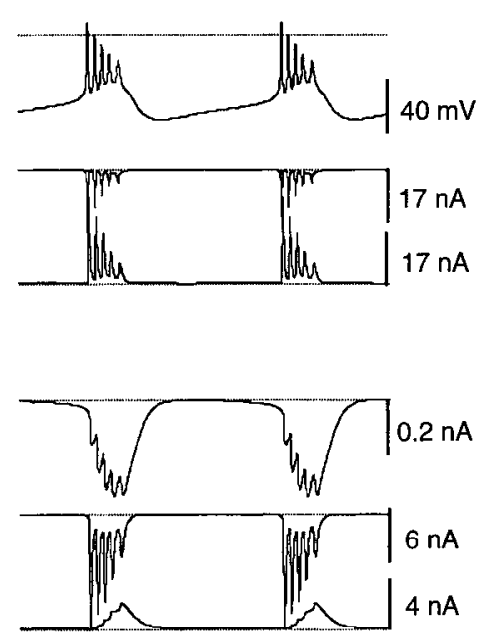

$1 \mathrm{nA}$
Figure 10. Currents flowing during activity in the model neuron. The $\bar{g}$ values wcre set so that the current amplitudes for the different states were as indicated in Figure 9. $A$, Inactivating spikes, $B$, Tonic firing. $C$, Bursting. The $\bar{g}$ values in $\mu S$ for $A-C$ were, respectively, $\bar{g}_{\mathrm{Nat}}=33,70,70 ; \bar{g}_{\mathrm{K} d}=70$, 47,$23 ; \bar{g}_{\text {Nat }}=0.23,0.46,0.46 ; \bar{g}_{\mathrm{Ca}}=$ $0.12,0.33,0.67 ; \bar{g}_{\mathrm{KCa}}=133,67,30 ; \bar{g}_{\mathrm{A}}$ $=11,7.3,3.6 ; \bar{g}_{\text {As }}=4,2.6,1.3 ; \bar{g}_{h}=$ $0.023,0.023,0.023$. $V$, voltage. For voltage trace, dashed line indicates 0 $\mathrm{mV}$; for current traces, dashed lines indicate 0 current. $I_{d c}$, DC current injection. 


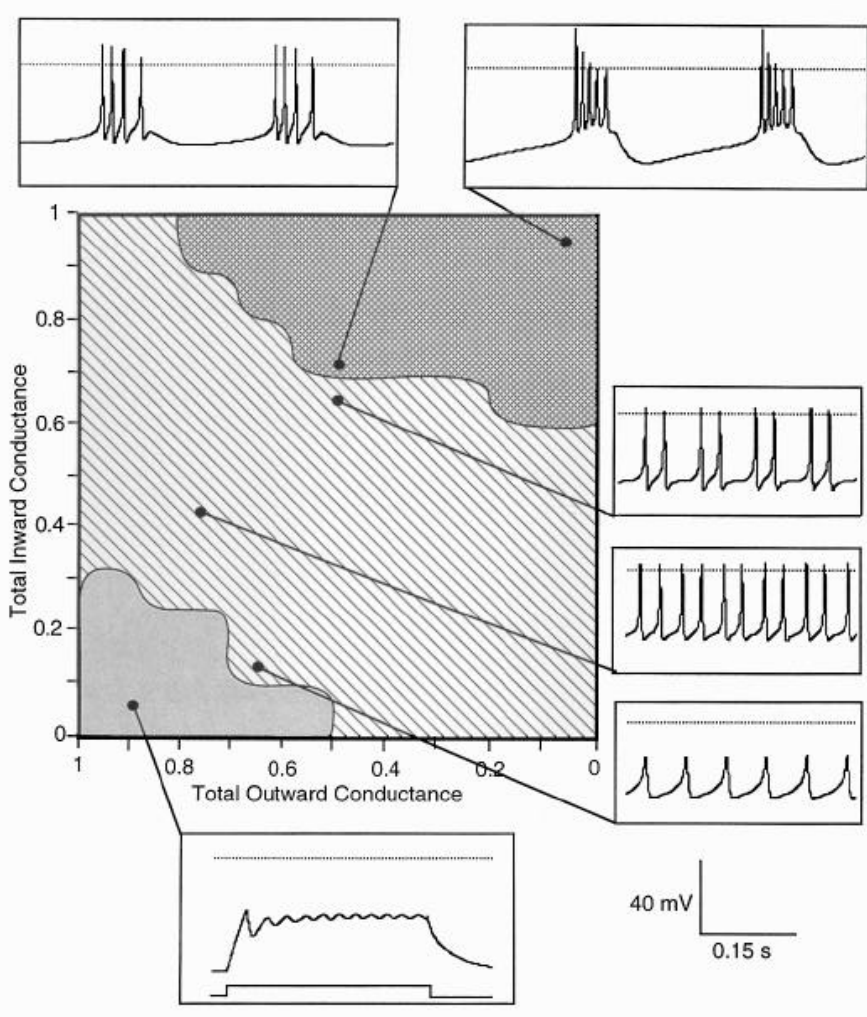

Figure 11. Activity of the model neuron in different conductance regimes. The total inward conductance is plotted on the y-axis $\left(\bar{g}_{\mathrm{Na}}+\bar{g}_{\mathrm{Nap}}\right.$ $\left.+\bar{g}_{\mathrm{Ca}}\right)$ and the total outward conductance is plotted on the $\mathrm{x}-\mathrm{axis}\left(\bar{g}_{\mathrm{Kd}}\right.$ $+\bar{g}_{\mathrm{As}}+\bar{g}_{\mathrm{A}}+\bar{g}_{\mathrm{K} d}$ ). For each conductance the difference between the $\bar{g}$ for inactivating spikes and the $\bar{g}$ for bursting was calculated and divided into 10 equal increments (values reported in Fig. 10 legend). Inward $\bar{g} \mathrm{~s}$ were varied together incrementally, with 0 being the starting values and 1 the ending values. Outward $\bar{g} \mathrm{~s}$ were varied together incrementally, with 1 being the starting values and 0 the ending values. Other conductances $\left(\bar{g}_{b}, \bar{g}_{l}\right)$ were held constant. The stippled area indicates regions in which the model neuron produced inactivating spikes; the hatched area indicates regions in which it produced tonic firing; and the crosshatched area indicates regions in which it produced bursts. Throughout these conductance regimes the model neuron was silent at rest. Examples of activity are from the regions indicated by the filled circles, and represent activity when the model neuron is depolarized. The dashed lines indicate $0 \mathrm{mV}$.

ing. This change in activity is accomplished by an increase in inward and decrease in outward current densities. Modeling studies demonstrate that these changes in current density can account for the changes in neuronal properties. STG neurons in culture therefore selectively adjust the magnitudes of their ionic conductances.

\section{Expression of conductances in culture}

The outward currents of STG neurons in the ganglion have been described for both the crab Cancer borealis and the spiny lobster Panulirus interruptus, and include $I_{\mathrm{KCa}}, I_{\mathrm{K} d}$, and $I_{\mathrm{A}}$ (Graubard and Hartline, 1991; Golowasch and Marder, 1992b). The outward currents expressed in culture are similar to those expressed in the ganglion. $I_{\mathrm{K} d}$ and $I_{\mathrm{KCa}}$ have similar voltage dependencies and pharmacological sensitivities in cultured STG neurons to those described for the crab LP neuron. $I_{\mathrm{A}}$ has both rapidly $\left(I_{\mathrm{A}}\right)$ and slowly inactivating $\left(I_{\mathrm{A} s}\right)$ components with similar voltage dependencies of activation and inactivation, but $I_{\mathrm{A} s}$ has a time constant of inactivation about an order of magnitude slower than $I_{\mathrm{A}}$. Cur- rents similar to $I_{\mathrm{A} s}$ have been described in a variety of neurons and contribute to long delays in firing and temporal integration (Storm, 1988; McCormick, 1991; Tokimasa et al., 1991; Nisenbaum et al., 1994). $I_{\mathrm{As}}$ can impart similar properties to STG neurons, and produces long-lasting excitability changes following strong depolarizations (Abbott et al., 1993; G. G. Turrigiano, L. F. Abbott, and E. Marder, unpublished observations).

We identified four inward currents in cultured STG neurons. $I_{h}$ has been well described previously, and we have not studied its properties further, except to note that every cultured STG neuron expressed $I_{h}$ to some degree. Sodium currents had been impossible to characterize previously in these neurons. We characterized two TTX-sensitive currents, a large, rapidly activating and inactivating current that resembles fast sodium currents described in many neurons, and a smaller persistent current similar to persistent sodium currents described in a variety of neurons (Yawo et al., 1985; Davis and Stuart, 1988; Llinás, 1988; French et al., 1990; Alzheimer et al., 1993). Finally, we have identified a high-threshold calcium current with both an inactivating and a persistent component, similar to the current partially characterized in the crab LP neuron.

With the currents described above blocked, little or no additional active current was observed in voltage-clamp experiments, and our model STG neuron with only these currents can reproduce very closely the different activity patterns seen in the biological neurons. This suggests that we have accounted for the major conductances expressed in culture. There is likely to be an additional array of conductances that are only active under the influence of neuromodulatory substances (e.g., Golowasch and Marder, 1992a).

\section{Accuracy of current measurements}

STG neurons have been difficult to voltage clamp in the ganglion, due to (1) the large capacitance; (2) the electrotonic distance of inward currents, especially fast sodium currents, from somatic recording sites; and (3) the difficulty of adequately removing presynaptic influences. In previous studies, the best clamp settling times achieved were on the order of $15-30 \mathrm{msec}$ Isolated, electrotonically compact neurons in culture have an average capacitance $(0.4-0.5 \mathrm{nF})$ much smaller than in the ganglion (10 $\mathrm{nF}$ or higher), allowing us to achieve clamp settling times of 1-10 msec. This allowed for reasonably accurate peak current measurements for most of the currents described here. The exception is $I_{\mathrm{Na}}$. We restricted our measurements of $I_{\mathrm{Na}}$ to neurons in which clamp settling times of $<1 \mathrm{msec}$ were achieved, but as $I_{\mathrm{Na}}$ is activating during this time our peak current measurements probably underestimate the actual values.

\section{Model neuron}

When the currents described above were incorporated into a conductance-based model STG neuron, the model reproduced each of the three activity states found in the biological neurons. In addition, the changes in current densities measured were sufficient to account for the changes in neuronal properties with time in culture. This is in contrast to developmental changes in neuronal properties, which in many cases depend upon changes in the kinetics or voltage dependence of currents (O'Dowd et al., 1988; Park and Ahmed, 1991; Lockery and Spitzer, 1992; Skaliora et al., 1993).

Bursting in the model neuron arises from a complex interaction between many currents. Bursts depend upon sodium and calcium currents, as do bursts in the biological neurons (Gola 
and Selverston, 1981; Harris-Warrick and Flamm, 1987; Turrigiano and Marder, 1993). Interestingly, bursts in the model neuron depend on $I_{\mathrm{Na}}$, as the strong depolarization due to $I_{\mathrm{Na}}$ is necessary to activate the higher-threshold $I_{\mathrm{Ca}}$. The separate contribution of $I_{\mathrm{Na}}$ and $I_{\mathrm{Nap}}$ to bursting in the biological neurons is unknown, as TTX blocks both currents with similar efficacy. In an earlier modeling study, a similar dependence of bursting on $I_{\mathrm{Na}}$ was observed in some conductance regimes (Epstein and Marder, 1990). An increase in $I_{\mathrm{Ca}}$ can render the model neuron bursts independent of sodium currents; under some modulated conditions STG neurons can also generate sodium-independent bursts (Harris-Warrick and Flamm, 1987), suggesting that these modulators are increasing the contribution of calcium currents to burst generation.

The model makes several simplifying assumptions that may be inaccurate. First, because the cultured neurons are electrotonically compact relative to neurons in the ganglion, we have used a single compartment model. It is unclear at the moment to what extent cultured neurons segregate conductances, but other crustacean neurons have been shown to differentially distribute inward and outward currents in culture (Meyers, 1993). Second, because the dynamics of calcium influx and buffering are completely unknown, we have modeled calcium buffering as a single exponential decay. The dynamics of intraccllular calcium are undoubtedly important in determining how $I_{\mathrm{Ca}}$ and $I_{\mathrm{KCa}}$ interact, so in future work it will be important to measure these parameters accurately. Finally, we have used voltage-clamp data from unidentified STG neurons, while the kinetics of a given current may vary between cell types. Variations in the kinetics of $I_{\mathrm{Ca}}$ and $I_{\mathrm{KCa}}$ in the model neuron, for example, can turn fast pyloriclike bursts into slower gastric-like bursts.

\section{Change in intrinsic properties with time in culture}

When isolated from modulatory and synaptic influences in the ganglion, STG neurons (with the possible exception of the pacemaker $A B$ neuron) fire tonically rather than in bursts. STG neurons have similar properties after $2 \mathrm{~d}$ in culture, but after an additional day become capable of firing in bursts both when depolarized and on the rebound, and appear to stabilize in this state. This sequence of events is very similar to the developmental sequence undergone by some vertebrate neurons with complex intrinsic properties (Gruol et al., 1992; Ramoa and McCormick, 1994).

This change in clectrical properties is accompanied by a change in the level of expression of conductances. Each class of current has a different pattern of expression with time in culture. Sodium current densities reach peak values and stabilize by $2 \mathrm{~d}$ in culture, whereas calcium currents increase significantly in density between 2 and $3 \mathrm{~d}$. Part of this increase may be explained by addition of membrane to fine neurites, which express inward currents in the ganglion. However, even neurons that did not regrow fine neurites were capable of firing action potentials and bursting, indicating that inward currents in culture can be added to somatic membrane, as has been described for other neurons following axotomy (Gilly and Brismar, 1989). At the same time that inward current densities increased, outward current densities decreased to about one-third of initial values, while there was no significant change in the average neuronal capacitance. Therefore, even if new membrane added in culture expressed only inward currents, the decrease in outward current density could not be accounted for by growth. This decrease in outward current density must reflect an active down regulation of outward currents.

Why do these neurons adjust the balance of their conductances in culture? Several factors may contribute to this change in intrinsic properties, including changes in trophic influences, changes due to axotomy and regrowth, and changes in synaptic drive. These factors are known to induce changes in a number of neuronal properties, including receptor or gene cxpression, the magnitude of individual ionic conductances, and the level of excitability of neurons (Favaron et al., 1992; Franklin et al. 1992; Stewart and Atwood, 1992; Traynor et al., 1992; HyattSachs et al., 1993).

Recent theoretical and experimental work noted that activity might play an important role in regulating the magnitude of neuronal conductances (Alkon, 1984; Franklin et al., 1992; Abboll and LeMasson, 1993; LeMasson et al., 1993; Garcia et al., 1994; Turrigiano et al., 1994). In the animal STG neurons normally fire in bursts, due to modulatory and synaptic drive. An interesting possibility is that these rhythmic inputs play a role in adjusting the magnitude of ionic conductances, so that when isolated from this drive STG neurons increase calcium currents and decrease outward currents to increase their intrinsic excitability. Recent experimental work suggests that such a mechanism operates in cultured STG neurons. Patterned stimulation that approximates the normal rhythmic drive of STG neurons, when delivered to bursting neurons in culture, reverses the transition from bursting to tonic firing (Turrigiano et al., 1994). These data suggest that one of the factors contributing to the change in expression of conductances in culture is the loss of synaptic drive, and that activity is able to regulate the balance of inward and outward conductances in these neurons.

\section{References}

Abbott LF, LeMasson G (1993) Analysis of neuron models with dynamically regulated conductances. Neural Comput 5:823.

Abbott LF, 'lurrigiano GG, Marder E (1993) A slowly inactivating potassium conductance underlies depolarization-induced transitions between tonic and bursting behavior in real and model neurons. Soc Neurosci Abstr 19:995.

Alkon DL (1984) Calcium-mediated reduction of ionic currents: a biophysical memory trace. Science 226:1037-1045.

Alzheimer C, Schwindt PC, Crill WE (1993) Modal gating of sodium channels as a mechanism of persistent sodium current in pyramidal neurons from rat and cat sensorimotor cortex. J Neurosci 13:660673

Buchholtz F, Golowasch J, Epstein IR, Marder E (1992) Mathematical model of an identified stonalogastric ganglion neuron. J Neurophysiol 67:332-340.

Canavier CC, Clark JW, Byrne JH (1991) Simulation of the bursting activity of neuron R15 in aplysia: role of ionic currents, calcium balance, and modulatory transmitters. J Neurophysiol 66:2107-2124.

Davis RE, Stuart AE (1988) A persistent, TTX-sensitive sodium current in an invertebrate neuron with neurosecretory ultrastructure. $J$ Neurosci 8:3978-3991.

Epstein IR, Marder E (1990) Multiple modes of a conditional neural oscillator. Biol Cybern 63:25-34.

Favaron M, Rimland JM, Manev H (1992) Depolarization and agonistregulated expression of neuronal metabotropic glutamate receptor 1 (mGluR1). Life Sci 50:PL189-PL194.

Franklin JL, Fickbohm DJ, Willard AL (1992) Long-term regulation of neuronal calcium currents by prolonged changes of membrane potential. J Neurosci 12:1726-1735.

French CR, Sah P, Buckett KJ, Gage PW (1990) A voltage-dependent persistent sodium current in mammalian hippocampal neurons. J Gen Physiol 95:1139-1155.

Garcia DE, Cavelié A, Lux HD (1994) Enhancement of voltage-gated $\mathrm{Ca}^{2+}$ currents induced by daily stimulation of hippocampal neurons with glutamate. J Neurosci 14:545-553. 
Gilly W, Brismar T (1989) Properties of appropriately and inappropriately expressed sodium channels in squid giant axon and its somata. J Neurosci 9:1362-1374.

Gola M, Selverston $\Lambda \mathrm{I}$ (1981) Ionic requirements for bursting activity in lobster stomatogastric neurons. J Comp Physiol 145:191-207.

Golowasch J, Marder E (1992a) Proctolin activates an inward current whose voltage dependence is modified by extracellular $\mathrm{Ca}^{2+}$. J Neurosci $12: 810-817$.

Golowasch J, Marder E (1992b) Ionic currents of the lateral pyloric neuron of the stomatogastric ganglion of the crab. J Neurophysiol $67: 318-331$.

Golowasch J, Buchholtz F, Epstein IR, Marder E (1992) Contribution of individual ionic currents to activity of a model stomatogastric ganglion neuron. J Neurophysiol 67:341-349.

Graubard K, Hartline DK (1991) Voltage clamp analysis of intact stomatogastric neurons. Brain Res 557:241-254.

Gruol DL, Deal CR, Yool AJ (1992) Developmental changes in calcium conductances contribute to the physiological maturation of cerebellar Purkinje neurons in culture. J Neurosci 12:2838-2848.

Guckenheimer J, Gueron S, Harris-Warrick RM (1993) Mapping the dynamics of a bursing neurun. Philos Trans R Soc Lond [Biol] 341 : 345-359.

Harris-Warrick RM, Flamm RE (1987) Multiple mechanisms of bursting in a conditional bursting neuron. J Neurosci 7:2113-2128.

Harris-Warrick RM, Marder E, Selverston AI, Moulins M, eds (1992) Dynamic biological networks. Cambridge, MA: MIT Press.

Hille B (1992) Ionic channels of excitable membranes. Sunderland, MA: Sinauer.

Hodgkin AI, Huxley AF (1952) A quantitative description of membrane current and its application to conduction and excitation in nerve. J Physiol (Lond) 117:500-544.

Hyatt Sachs H, Schreiber RC, Bennett TA, Zigmond RE (1993) Phenotypic plasticity in adult sympathetic ganglion in vivo: effects of deafferentation and axotomy on the expression of vasoactive inteslinal peptide. J Neurusci 13:1642-1653.

Kaczmarek LK, Levitan IB (1987) Neuromodulation: the biochemical control of neuronal excitability. New York: Oxford UP.

Kiehn O, Harris-Warrick RM (1992) Serotonergic modulation of a hyperpolarization-activated cation current $\left(I_{i}\right)$ and a calcium-dependent outward current $\left(I_{0(\mathrm{Ca})}\right)$ in an identified crustacean motoneuron. J Neurophysiol 68:496-508.

Koch C, Segev I, eds (1989) Methods in neuronal modeling: from synapses to networks. Cambridge, MA: MIT Press.

LeMasson G, Marder E, Abbott LF (1993) Activity-dependent regulation of conductances in model neurons. Science 259:1915-1917.

Llinás RR (1988) Intrinsic electrophysiological properties of mammalian neurons: insights into central nervous system function. Science $242: 1654-1664$.

Lockery SL, Spilzer NC (1992) Reconstruction of action potential de- velopment from whole-cell currents of differentiating spinal neurons. J Neurosci 12:2268-2287.

McCormick DA (1991) Functional properties of a slowly inactivating potassium current in guinea pig dorsal lateral geniculate relay neurons. J Neurophysiol 66:1176-1189.

Meyers DER (1993) Distribution of ionic currents in the soma and growing region of an identified peptidergic neuron in defined culture. J Neurophysiol 69:406-415.

Nisenbaum ES, Xu ZC, Wilson CJ (1994) Contribution of a slowly inactivating potassium current to the transition to firing of neostriatal spiny projection neurons. J Neurophysiol 71:1174-1189.

O'Dowd DK, Ribera AB, Spitzer NC (1988) Development of voltagedependent calcium, sodium, and potassium currents in Xenopus spinal neurons. J Neurosci 8:792-805.

Panchin YV, Arshavsky YI, Selverston AI, Cleland TA (1993) Lobster stomatogastric neurons in primary culture. I. Basic characteristics. J Neurophysiol 69:1976-1992.

Park CC, Ahmed Z (1991) Characterization of sodium current in developing rat diencephalic neurons in serum-free culture. $\mathfrak{J}$ Neurophysiol 65:1011-1021.

Ramoa AS, McCormick DA (1994) Developmental changes in electrophysiological properties of LGNd neurons during reorganization of retinogeniculate connections. J Neurosci 14:2089-2097.

Rinzel J, Lee YS (1987) Dissection of a model for neuronal parabolic bursting. J Math Biol 25:653-675.

Skaliora I, Scopby RP, Chalupa LM (1993) Prenatal development of excitability in cat retinal ganglion cells: action potentials and sodium currents. J Neurosci 13:313-323.

Stewart BA, Atwood HL (1992) Synaptic plasticity in a regenerated crayfish phasic motoneuron. J Neurobiol 23:881-889.

Storm JF (1988) Temporal integration by a slowly inactivating $\mathrm{K}^{+}$ current in hippocampal neurons. Nature 336:379-381.

Tierney AJ, Harris-Warrick RM (1992) Physiological role of the transient potassium current in the pyloric circuit of the lobster stomatogastric ganglion. J Neurophysiol 67:599-609.

Tokimasa T, Tsurusaki M, Akasu T (1991) Slowly inactivating potassium current in cultured bull-frog primary afferent and sympathetic neurons. J Physiol (Lond) 435:585-604.

Traynor P, Dryden WF, Smith PA (1992) Trophic regulation of action potential in bullfrog sympathetic neurons. Can J Physiol Pharmacol 70:826-834.

Turrigiano GG, Marder E (1993) Modulation of identified stomatogastric ganglion neurons in primary cell culture. J Neurophysiol 69: 1993-2002.

Turrigiano GG, Abbott LF, Marder E (1994) Activity changes the intrinsic properties of cultured neurons. Science 264:974-976.

Yawo H, Kojima H, Kuno M (1985) Low-threshold, slow-inactivating sodium potentials in the cockroach giant axon. J Neurophysiol 54: $1087-1100$ 